G18.95-1.1, A COMPOSITE SUPERNOVA REMNANT INTERACTING WITH THE AMBIENT INTERSTELLAR MEDIUM

E. Fürst, W. Reich, E. Hummel

Max-Planck-Institut für Radloastronomie

Auf dem Hügel 69, D-5300 Bonn 1, F.R.G.

\title{
Y. Sofue
}

Department of Astronomy, Faculty of Science

University of Tokyo, Bunkyo-ku, Tokyo 113, Japan

\begin{abstract}
New radio continuum and spectral line observations of the Galactic radio source $\mathrm{G18.95-1.1}$ are reported. The distance to $\mathrm{G} 18.95-1.1$ is $2 \mathrm{kpc}$ as derived from HI-21 cm spectral line observations. These data also indicate an interaction with the interstellar medium. The radio continuum observations classify G18.95-1.1 as a composite supernova remnant.
\end{abstract}

The Galactic radio source G18.95-1.1 was found to be nonthermal by Fürst et al. (1985). The radio spectrum of its integrated flux density between $57.5 \mathrm{MHz}$ and $5 \mathrm{GHz}$ is now well established $(\alpha=-0.28 \pm 0.05$, $\mathrm{S}_{\nu} \sim \nu^{\alpha}$, Odegard, 1986). From radio continuum maps (see Fig. 1 in the paper by Fürst et al., 1985) it is apparent that G18.95-1.1 is composed of two components. A large-scale diffuse emission (Fig. 1a), which contains $79 \%$ of the total flux density, peaks near the geometrical centre of
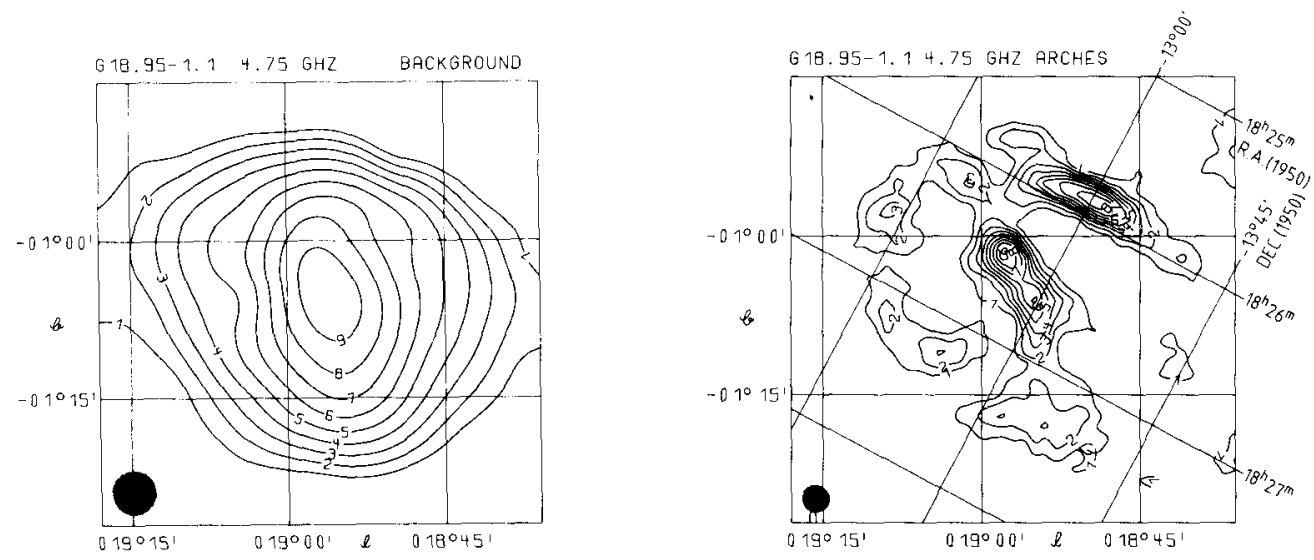

Figure 1: (a) Contour plot of the diffuse, centrally peaked component of G18.95-1.1 at $4.75 \mathrm{GHz}$. Contour steps are in $20 \mathrm{mJy} / 2.45$ beam. The map is convolved to a HPBW of 4.2 as is indicated by the hatched circle.

(b) Contour plot of the arc component of G18.95-1.1 at $4.75 \mathrm{GHz}$. Contour steps are $20 \mathrm{mJy} / 2.45$ beam. The HPBW is $2: 45$ as is indicated by the hatched circle. 
the source. Superposed are various arc-like features (Fig. 1b) containing $21 \%$ of the total flux density. The most prominent arc features are shown in Fig. 2 at an angular resolution of $19^{\prime \prime} \times 14^{\prime \prime}\left(p . a .=0^{\circ}\right)$ at 1.5 and 4.9 GHz. The observations were made with the Very Large Array (VLA) in its

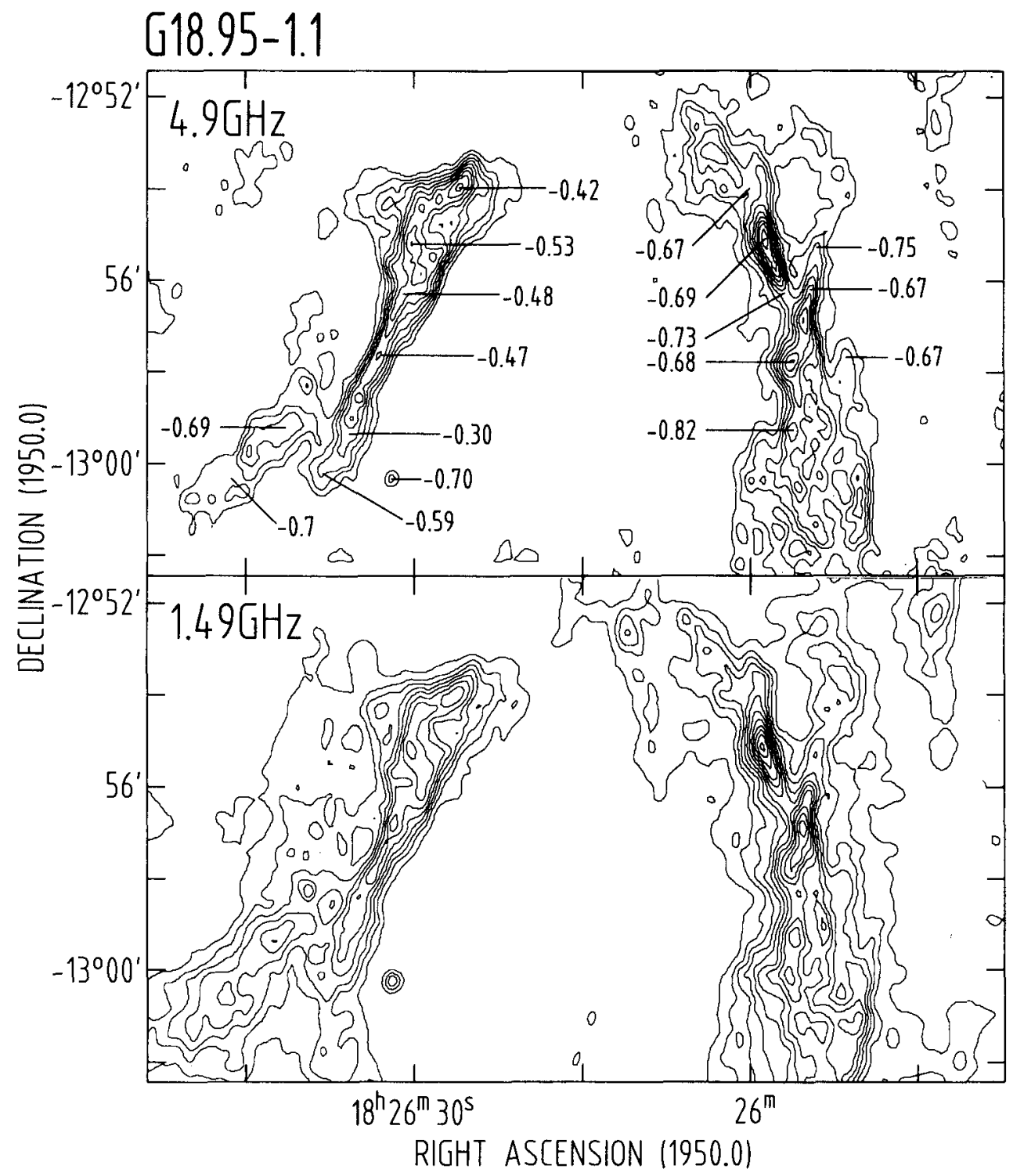

Figure 2: Contour plot of a part of G18.95-1.1 at $4.9 \mathrm{GHz}$ (upper panel, contour steps $0.5 \mathrm{mJy} /$ beam) and $1.49 \mathrm{GHz}$ (lower panel, contour steps $1 \mathrm{mJy} /$ beam) obtained with the Very Large Array ( $\mathrm{C}$ and $\mathrm{D}$ configuration). Both maps are corrected for the primary beam attenuation. The HPBW is $19^{\prime \prime} \times 14^{\prime \prime}$. In the upper panel some spectral indices $\alpha\left(S_{\nu}-\nu^{\alpha}\right)$ are quoted. 
$D$ and $C$ configurations. We also derived the spectral index between the two frequencies at some selected positions. These spectral indices have to be considered as lower limits (the spectra are probably flatter): due to missing short spacings we miss slightly more structure at $4.9 \mathrm{GHz}$. Taking also into account single-dish observations the spectral index of the central feature of $\mathrm{G} 18.95-1.1$ is $\alpha \approx-0.4 \pm 0.2$, i.e. similar to the spectral index of the integrated emission. On the other hand, all other arc-like structures visible in Fig. 1b have relatively steep spectra $(\alpha=-0.65 \pm 0.2)$. This spectral index is typical for shell-type supernova remnants (SNRs). These steep spectral arcs and the flat spectrum, large-scale component classify G18.95-1.1 as a composite SNR (Weiler, 1983). Composite SNRs are expected to be powered by central activity. The flat spectrum central feature may be the signature of the outflow of relativistic electrons from this central activity feeding the large-scale component. The relativistic electrons are confined by the SNR-shock interacting with the interstellar medium. The steep spectrum arcs represent these shocks.

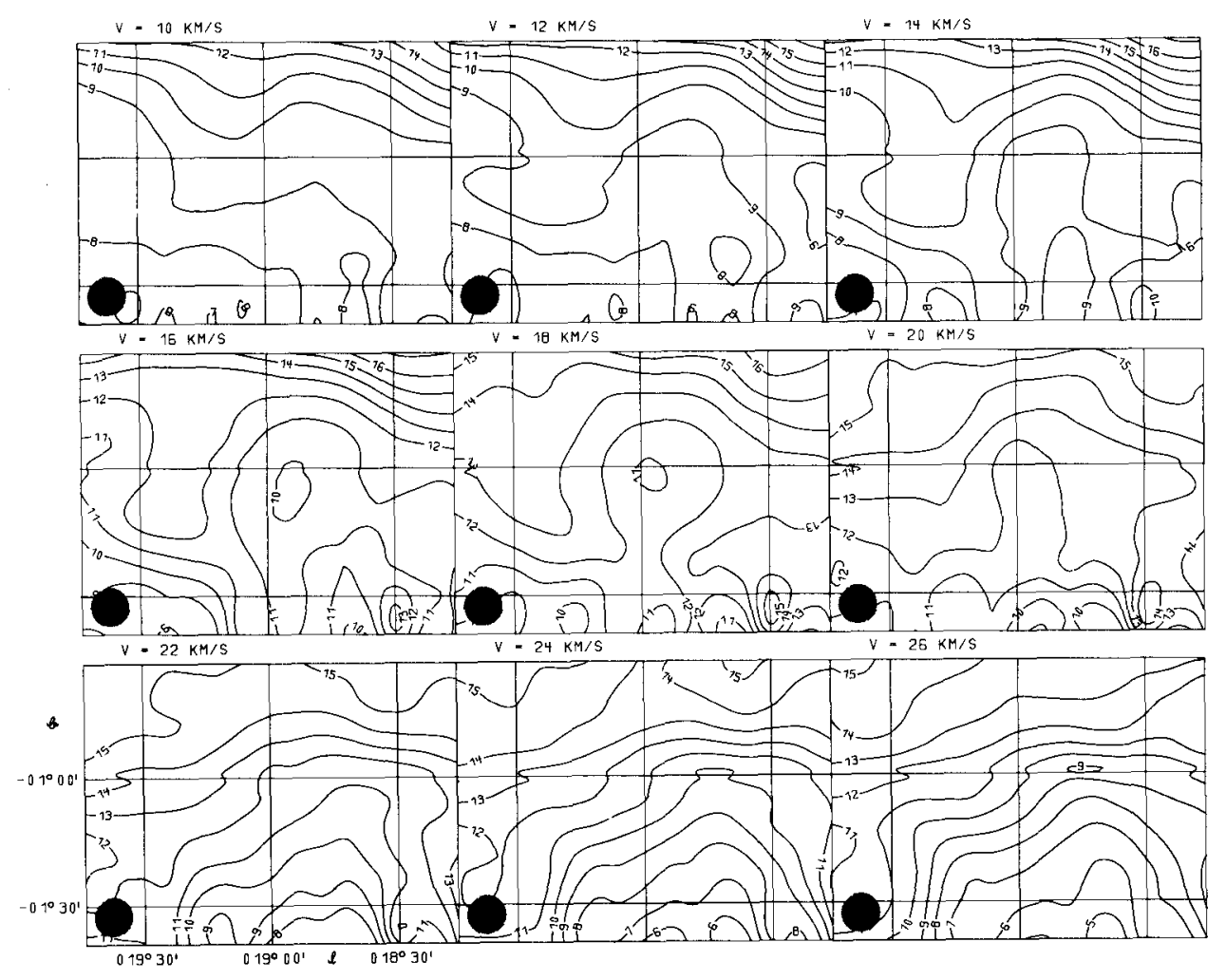

Figure 3: Contour plots of the $\mathrm{HI}-21 \mathrm{~cm}$ line antenna temperature obtained with the Effelsberg 100-m telescope. The HPBW is $9^{\prime}$ as is indicated by the hatched circles. Plots are shown at radial velocities between $10 \mathrm{~km} \mathrm{~s}^{-1}$ and $26 \mathrm{~km} \mathrm{~s}^{-1}$ at an interval of $2 \mathrm{~km} \mathrm{~s}^{-1}$. Contour steps are $5 \mathrm{~K} \mathrm{~T}_{\mathrm{A}}$ beginning at $2 \mathrm{~K} \mathrm{~T}$ (contour 1 ). 
The interaction of G18.95-1.1 with the interstellar medium is supported by $\mathrm{HI}-21 \mathrm{~cm}$ line observations made with the $100-\mathrm{m}$ telescope by Braunsfurth and Rohlfs (1984). In Fig. 3 we show channel maps between the velocity $V_{L S R}=10 \mathrm{~km} \mathrm{~s}^{-1}$ and $V_{L S R}=26 \mathrm{~km} \mathrm{~s}^{-1}$. A strong depression in the HI-antenna temperature is visible close to the geometrical centre of G18.95-1.1 at a velocity of $V_{L S R}=18 \mathrm{~km} \mathrm{~s}^{-1}$. The discussion of the depression by Fürst et al. (1987) shows that it is most likely explained by missing HI-gas across the SNR. The association of the depression with G18.95-1.1 sets limits for the distance to the SNR. Common rotation models give $2 \mathrm{kpc}$ or $15 \mathrm{kpc}$ on the far side of the Galaxy. It can be shown that the large distance is unprobable: G18.95-1.1 would be much more energetic than the Crab nebula. Whence, the distance is $2 \mathrm{kpc}$ (Sagittarius arm). The missing column density across G18.95-1.1 at the centre of the $\mathrm{HI}$-depression is $\langle\Delta N\rangle=9.10^{19} \mathrm{~cm}^{-2}$, which scales to $\mathrm{N}=2 \mathrm{~cm}^{-3}$ at a distance of $2 \mathrm{kpc}$ (linear size of the SNR $\approx 20 \mathrm{pc}$ ). Therefore, G18.95-1.1 is probably expanding into an ambient medium of density $\mathrm{N} \gtrsim 2 \mathrm{~cm}^{-3}$. The low angular resolution of the $21 \mathrm{~cm}$ line data obtained with the $100-\mathrm{m}$ telescope is not sufficient to investigate the interaction conclusively. In particular, the question whether the depression is caused by the SNR ejecta/shock or by the stellar wind of the progenitor star cannot be answered, although the low velocity dispersion of the depression $\left(\Delta \mathrm{V} \approx 8 \mathrm{~km} \mathrm{~s}^{-1}\right.$ ) argues in favour of the latter. More observations in $\mathrm{HI}$ and in molecular lines with better angular resolution are needed to study G18.95-1.1 as one of the rare cases, where a clear interaction of an SNR with the ambient interstellar medium is visible.

A detailed analysis of the new observations is in preparation (Fürst et al., 1987).

\section{References}

Braunsfurth, E., Rohlfs, K.: 1984, Astron. Astrophys. 57, 189

Fürst, E., Reich, W., Reich, P., Sofue, Y., Handa, T.: 1985, Nature 314, 720

Fürst, E. et al.: 1987, in preparation

Odegard, N.: 1986, Astron. J. 92, 1372

Weiler, K.W.: 1983, The Observatory, 1054th edition 